\title{
Quantitative Analysis of Sulfur in Polymer Materials.
}

\author{
Stuart McKernan ${ }^{1}$, Mary I. Buckett ${ }^{1}$ and William G. Stratton ${ }^{1}$ \\ 1. 3M Corporate Research Analytical Laboratory, 3M Center, Building 201-BE-16, St. Paul, MN 55144
}

Energy Dispersive Analysis (EDS) in the TEM relies on being able to detect X-rays produced by the different atomic species within a sample as it is irradiated by the incident electron beam. A number of factors affect how the number of X-rays of each species that are detected relate to the actual chemical composition of the sample; in particular the proportions of X-rays that are absorbed in the specimen and surrounding environment between being generated and being detected and analyzed will have a significant effect on the final composition reported. Typically for light elements in an irregular sample the proportion of detected versus generated is an unknown but very influential quantity, leading to quantification values with large error bars. In this paper we report our efforts to determine optimal criteria to obtain reasonable values for the Sulfur:Carbon ratio and the minimum detectable concentration for Sulfur in these polymer materials.

The most accurate composition determinations are obtained from analyses using known standards under the same analytical conditions as the unknown material. This is more accurate than calculating the compositions using the as-provided k-factors and standard correction factors in a "standardless analysis" [1]. To that end standard spectra from a samples of known composition (using as close to ideal acquisition parameters as possible) were created. Test samples were prepared in the form of cured epoxy with various loadings of Jeffamine Sulfonate (JAS). This produced a set of homogeneous S-containing standards. The nominal concentration of JAS in the epoxy for each sample was designed as 5\%, 10\%, and $20 \%$. The actual concentrations of $\mathrm{S}$ in the test samples were determined by halogen analysis by combustion ion chromatography as: $0.22,0.50$, and $0.98 \mathrm{wt} \%$ respectively. TEM samples were prepared from the JAS/epoxy by ultramicrotomy to produce thin, parallel-sided sections 100nm thick that were supported on an ultrathin carbon film. The TEM samples were then analyzed in a JEOL 2100F operating at 200kV using a Thermo Scientific Noran EDS System.

Sample homogeneity and beam-stability were examined by comparing multiple spectra from different areas of the samples, or the same area during multiple scans. The carbon content of the TEM support film was subtracted from each active spectra by acquiring spectra from an adjacent region of the support film alone for the same time as the actual film spectrum and then subtracting the two spectra. In figure 1, four adjacent areas on a section of the $20 \%$ JAS film are outlined and were analyzed separately and together. They show some distinct variations in composition, together with a bright damaged spot. In figure 2, results of multiple analyses from the same area indicate that after the first scan, where the oxygen signal appears to decrease slightly, the signals (and particularly the $S$ content) appear to remain stable during the multiple EDS scans. For the standard spectrum, using the highest S-content (20 wt $\%$ JAS) TEM sample, multiple spectra were acquired for 1000 s from different areas. Those summed spectrum were then used to define the experimental $\mathrm{C}$ and S k-ratios in the NSS 3.0 software. Additional spectra from the $20 \%$ JAS content and the $10 \%$ and $5 \%$ content were then quantified using these ratios. Comparisons between the EDS results and the Halogen Analysis are shown in figure 3.

Using this standards-based analysis reasonably good agreement between the EDS composition and that obtained by halogen analysis was obtained for the $20 \%$ JAS sample (corresponding to a sulfur content of 
$\sim 1$ wt \%). The samples appeared to be sufficiently robust under the electron beam to produce consistent composition analysis even with $1000 \mathrm{~s}$ dwell times. Both the $20 \%$ JAS and 10\% JAS samples produced samples with detectable $\mathrm{S}$ content at the $1 \mathrm{wt} \% \mathrm{~S}$ and $0.5 \mathrm{wt} \% \mathrm{~S}$ level. The $5 \%$ JAS produced a sample with $0.22 \mathrm{wt} \% \mathrm{~S}$ that is below the detection limit by EDS under these conditions. [2]

References:

[1] D B Williams and C B Carter "Transmission Electron Microscopy", (Plenum, New York) 1996. [2] The authors acknowledge Jay Lomeda of the Corporate Research Materials Lab at 3M for the preparation of the samples used in this work.

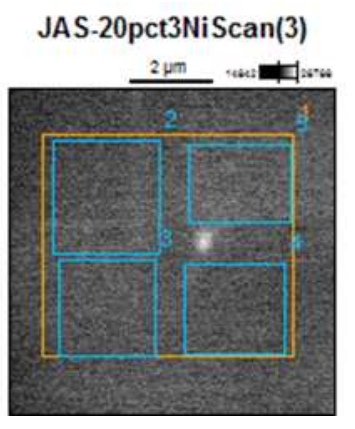

\begin{tabular}{|l|r|c|c|}
\hline Scan area & $\boldsymbol{C}-\boldsymbol{K}$ & $\boldsymbol{O}-\boldsymbol{K}$ & $\boldsymbol{S}-\boldsymbol{K}$ \\
point1 & 95.150 & 3.981 & 0.868 \\
point2 & 97.305 & 2.464 & 0.231 \\
point5 & 98.046 & 1.555 & 0.398 \\
point3 & 97.205 & 2.408 & 0.387 \\
point4 & 96.572 & 2.409 & 1.019 \\
\hline
\end{tabular}

Figure 1. Four adjacent areas outlined in blue analyzed independently, and together outlined in orange

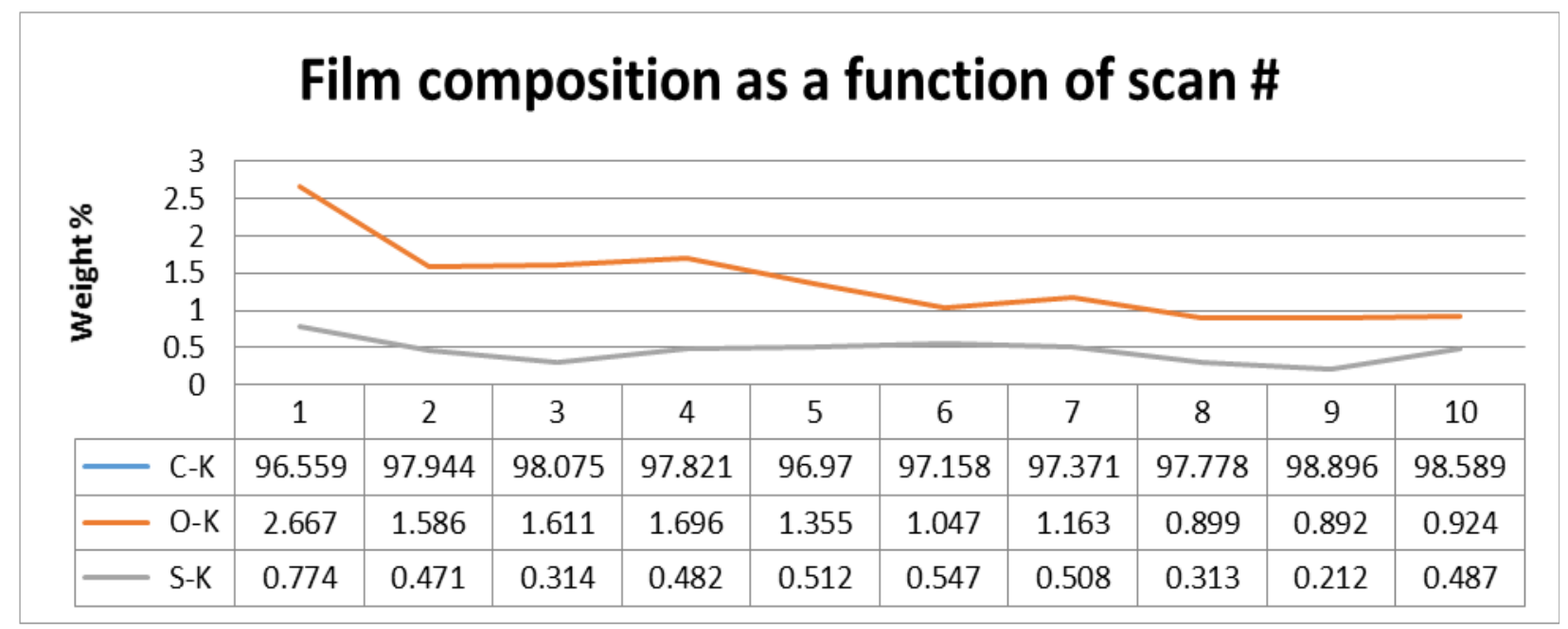

Figure 2. Plot of the concentration of $\mathrm{O}$ and $\mathrm{S}$ as a function of scan number from the same area.

\begin{tabular}{c|l|l|l|l}
\hline Sample & $\begin{array}{l}\text { Sulfur }(\text { wt } \%) \\
\text { Halogen Analysis }\end{array}$ & $\begin{array}{l}\text { Sulfur }(\text { wt\% }) \\
\text { (S)TEM EDS }\end{array}$ & $\begin{array}{l}\text { Detectable by (S)TEM } \\
\text { EDS Analysis }\end{array}$ & $\begin{array}{l}\text { Standardless EDS value } \\
\text { (for comparison) }\end{array}$ \\
\hline $5 \%$ JAS & $0.22 \pm 0.001$ & $0.06 \pm 0.04$ & No & $0.015 \pm \sim 5.0$ \\
$10 \%$ JAS & $0.50 \pm 0.002$ & $0.75 \pm 0.47$ & Yes & $0.164 \pm \sim 5.0$ \\
$20 \%$ JAS & $0.98 \pm 0.02$ & $0.86 \pm 0.25$ & Yes & $0.159 \pm \sim 5.0$ \\
\hline
\end{tabular}

Figure 3. Comparison of cured epoxy blocks with different JAS content. 